\title{
Escala de Calidad de Vida en Usuarios de Drogas Inyectadas (IDUQoL): Valoración psicométrica de la versión española
}

\author{
Ioseba Iraurgi Castillo \\ Módulo de Asistencia Psicosocial de Rekalde (Psicólogo Clínico) \\ Dirección de Drogodependencias - Gobierno Vasco (Consultor) \\ Enviar correspondencia a: \\ loseba Iraurgi Castillo. Módulo de Asistencia Psicosocial de Rekalde. Camilo Villabaso 24 lonja - 48002 Bilbao \\ e-mail: iraurgi@telefonica.net
}

Recibido: Febrero de 2008 Aceptado: Abril de 2008

\section{RESUMEN}

Antecedentes: La valoración de la calidad de vida se ha convertido en el área de la investigación clínica en uno de los más utilizados indicadores de resultados. Para ello, se precisa de instrumentos adaptados y validados al contexto donde han de utilizarse. Objetivo: Analizar las características psicométricas del IDUQoL (Injection Drug User Quality of Life Scale) en su adaptación a población española. Método: Participan en el estudio 100 personas diagnosticadas de adicción a opiáceos incluidas en un programa de mantenimiento con metadona, quienes responden al IDUQoL -en su versión de 21 dominiosy otros dos instrumentos de calidad de vida -el SF-36 y EQ-5D-. Resultados: Se obtienen datos de fiabilidad óptimos (alpha de Cronbach de 0,92 y coeficiente de correlación Intraclase para el test-retest de 0,79). Análisis factoriales exploratorios y confirmatorios y el análisis de la matriz de correlaciones semi-parciales concluyen la unidimensionalidad del constructo con una varianza explicada del $43,8 \%$ e índices de ajuste adecuados $(\mathrm{GFI}=0,92 ; \mathrm{CFI}=0,97 ; \mathrm{RMSEA}=0,044)$. Asimismo, se obtienen datos de validez convergente y de criterio muy adecuados (coeficientes de correlación con el SF-36 y EQ-5D entre 0,34 y 0,61 , y con otras variables criterio entre 0,23 y 0,$39 ; p<0,05$ ), si bien el análisis de la validez discriminante no es del todo concluyente. Conclusiones: Los resultados obtenidos presentan el IDUQoL como un instrumento específico de calidad de vida en el ámbito de los trastornos adictivos con adecuadas características en su adaptación al español.

Palabras clave: Calidad de vida, adaptación de escalas, características psicométricas, validación, fiabilidad, usuarios de drogas.

\section{ABSTRACT}

Background: The assessment of quality of life has become one of the priority indicators of outcomes in the field of clinical research. Therefore, it is necessary to use instruments adapted to and validated for the context in which they are to be used. Purpose: To assess the psychometric properties of the IDUQoL (Injection Drug User Quality of Life Scale) in its Spanish adaptation. Method: Participants were 100 persons with a diagnosis of opiate addiction who were on a methadone maintenance programme. They responded to the IDUQoL -in its 21-domain version- and two other quality of life instruments (the SF-36 and the EQ-5D). Results: Good reliability data are obtained (Cronbach's alpha of 0.92 and Intraclass Correlation Coefficient for the test-retest of 0.79). Exploratory and confirmatory factor analyses and semi-partial correlations analysis confirm the unidimensionality of the construct, with explained variance of $43.8 \%$ and adequate fit indices $(\mathrm{GFI}=0.92 ; \mathrm{CFI}=0.97$; RMSEA $=0.044)$. Convergent and criterion-related validity were also quite adequate (correlation coefficients with the SF-36 and EQ-5D were situated between 0.34 and 0.61 , and with other criterion variables between 0.23 and 0.39 ; $p<0.05$ ), even though the discriminant validity analysis is not completely conclusive. Conclusion: The findings from this study provide evidence to support the use of the IDUQoL as a specific quality of life instrument in the addictive disorders context, and confirm its appropriate characteristics in the Spanish adaptation.

Key words: Quality of life, adaptation scales, psychometric properties, validation, reliability, drug user. 


\section{INTRODUCCIÓN}

E n las últimas décadas viene desarrollándose un cambio importante en las medidas de valoración de los resultados de salud debido en parte a varios factores. En primer lugar, el desarrollo de las tecnologías sanitarias y evaluación continuada de los protocolos de tratamiento han propiciado la diversificación y mayor especificidad de los recursos terapéuticos, de forma que se han mejorando las respuestas de salud de las personas enfermas, se ha reducido de forma muy importante la mortalidad y, por ende, prolongado los años de vida. Asociado a ello, enfermedades que antes propiciaban altos niveles de mortalidad se han tornado menos insidiosas presentando menos patología asociada y tornándose hacia una evolución crónica; tal es el caso del SIDA tras la aparición del tratamiento anti-retroviral de alta eficacia, o el caso de los trastornos por dependencia de opiáceos con la aplicación de los programas de metadona y las medidas de reducción de daños ${ }^{1-3}$.

Ya no basta con valorar si un tratamiento reduce determinada sintomatología frente a otro tratamiento estándar cuando ambos resultan de una eficacia similar. Ahora interesa conocer cuál de estos tratamientos incide menos negativamente en la vida de la persona tratada y, por ello, se prima la percepción subjetiva de la persona que recibe el tratamiento. A esta dimensión de valoración corresponde el importante auge que viene presentando la Calidad de Vida Relacionada con la Salud (CVRS) como indicador de resultados, aspecto éste que también ha sido valorado como adecuado en el área de los trastornos adictivos ${ }^{4-6}$.

La generación de instrumentos de CVRS ha resultado ser una empresa muy prolífica, habiéndose desarrollado hasta el momento más de medio millar ${ }^{7-11}$. Una gran parte de estos instrumentos de medida son cuestionarios de autoinforme que contienen instrucciones para su cumplimentación y cuyo objetivo es la medición de las dimensiones de la CVRS a través de un número variable de ítems descriptivos que componen los diferentes niveles de cada dimensión. Entre los diferentes criterios de clasificación de los instrumentos de medida de la CVRS, destacaremos, por su aceptación, el que distingue entre instrumentos genéricos e instrumentos específicos ${ }^{12-14}$.

Los instrumentos genéricos son denominados como tal por no estar relacionados con ningún tipo de enfermedad en particular. Cubren un amplio espectro de dimensiones de la CVRS (discapacidad, funcionamiento físico y social, percepción de bienestar físico y emocional, etc.) y han sido diseñadas para poder ser aplicadas tanto a la población general como a grupos específicos de pacientes y para evaluar una gran variedad de enfermedades y/o tratamientos.
Los instrumentos específicos se orientan a evaluar las dificultades que se presentan en un grupo específico de enfermos (p.ej.: la utilización del índice de Katz para población geriátrica) o en una determinada enfermedad (Cuestionario de calidad de vida en la diabetes). Sólo incluyen aquellos aspectos de la calidad de vida que son relevantes para la enfermedad a la que se aplican. Las medidas concretas se centran en áreas en las que los clínicos, a través de la entrevista con el paciente y la exploración física, se encuentran más familiarizados, o en las áreas que los propios enfermos refieren como mayormente afectadas ${ }^{14,15}$.

En el ámbito de los trastornos adictivos, la valoración de la calidad de vida ha sido mayormente realizada a través de la utilización de instrumentos genéricos, siendo los más ampliamente utilizados el SF-36, y otras versiones de la familia de instrumentos MOS-SF, y el NHP (Nottingham Health Profile). Para una revisión de los instrumentos de CVRS utilizados en trastornos adictivos véase los trabajos de Iraurgi ${ }^{15}$ y Morales-Manrique y colaboradores ${ }^{16}$. Por su parte, los instrumentos de CVRS específicos en drogodependencias han recibido una atención muy limitada conociéndose hasta el momento tan solo tres instrumentos: 1) el QoL-DA (Quality of Life Scale for Drug Addicts); 2) el IDUQoL (Injection Drug User Quality of Life Scale); y 3) el TECVASP (Test para la Evaluación de la Calidad de Vida en Adictos a Sustancias Psicoactivas). Del primero, creado por un grupo de investigadores chino, sólo se ha publicado el proyecto de desarrollo del instrumento ${ }^{17}$, no habiéndose encontrado otras presentaciones de resultados, quizá debido a que sólo se haya difundido en aquel país. El TECVASP es un instrumento específico generado en nuestro ámbito por Oscar Lozano y colaboradores $^{18,19}$, con muy buenas características psicométricas que valora hasta nueve áreas de la calidad de vida subsumidas en dos dominios generales (físico y psicosocial), presentándose como un instrumento muy prometedor en la valoración de la calidad de vida en drogodependencias.

Por su parte, el IDUQoL fue creado en Canadá como respuesta a la ausencia de instrumentos de calidad de vida suficientemente sensibles para captar los cambios en esta área en personas con problemas relacionados con el consumo de drogas. Las autoras del IDUQoL proponen el desarrollo del instrumento partiendo de la conceptualización que los Usuarios de Drogas Inyectadas (UDI) hacen de la calidad de vida a partir de su propia experiencia, tratando de captar las posibles variaciones existentes y ser capaz de responder a los cambios en la condiciones de vida de estas personas. En su desarrollo inicial ${ }^{20,}{ }^{21}$ se proponían 17 áreas o dominios de la calidad de vida, siendo incrementado el número de dominios a 21 en una versión posterior ${ }^{22}$. La IDUQoL fue utilizada en varios estudios y ofrecidos sus resultados en presentaciones a congresos internacionales ${ }^{23-26}$, pero no ha sido hasta 
el año 2005 que han aparecido los primeros datos de valoración psicométrica de su validez y fiabilidad con una muestra suficiente ${ }^{22}$. Los resultados obtenidos en este estudio muestran una buena adecuación psicométrica del instrumento. La consistencia interna del IDUQoL muestra un coeficiente alpha de Cronbach de 0,88 con una fiabilidad test-retest para el total de la escala de 0,78 con una variación entre 0,32 y 0,67 atendiendo al total de dominios. Un análisis factorial de componentes principales logró extraer un único factor que explicaba el 30,5\% de la varianza, y cuyo valor propio (eigenvalue $=6,40$ ) excedía por encima de 4 veces la ratio respecto al valor del segundo factor, siendo este un criterio para mantener la unidimensionalidad del constructo ${ }^{27-29}$. Los ítems componentes saturaban el factor único con cargas que oscilaban entre un valor de 0,31 y 0,71. Asimismo, se obtuvieron datos que avalaban una adecuada validez de criterio (puntuaciones bajas en el IDUQoL se asociaban con una precaria estabilidad del alojamiento, con consumo reciente de drogas, con haber sufrido sobredosis en los últimos seis meses, con prestar servicios sexuales, o con haber intercambiado jeringuillas) y adecuada validez discriminante y convergente (correlaciones de 0,59 con la escala de satisfacción con la vida y de 0,54 con la escala de autoestima de Rosemberg). En las conclusiones del estudio, las autoras proponen el IDUQoL como un instrumento adecuado y factible de utilización en la valoración de la calidad de vida de usuarios de drogas y en la evaluación de intervenciones con esta población.

En aras a poder contar con instrumentos específicos suficientes adaptados al castellano para la valoración de la calidad de vida en personas con problemas adictivos, el objetivo del presente estudio es presentar las características del IDUQoL y ofrecer datos de la valoración psicométrica de la versión española del instrumento.

\section{MATERIAL Y MÉTODOS}

\section{Muestra y participantes}

Se ha evaluado un total de 100 personas con diagnóstico de Trastorno por Dependencia de Opiáceos según criterios DSM-IV incluidos en un programa de mantenimiento con metadona. Para su captación, se utilizó un procedimiento de lista correlativa según se habían ido incorporando al programa y en función de dos criterios de selección hasta completar el número fijado a priori de 100 participantes. Ocho personas elegibles que cumplían criterios de inclusión, rehusaron participar en el estudio alegando falta de tiempo (2), falta de ganas (5) o porque 'ya estaba harto de que le usaran como conejillo de indias' (1). El resto de participantes $(n=100)$ accedieron a colaborar en el estudio previa información de los objetivos y dando consentimiento de forma verbal.

Los criterios de selección, dados los objetivos de un estudio más amplio, consistían en que el 50\% fueran seropositivos al Virus de Inmunodeficiencia Humana (VIH), y al menos en otro $50 \%$ de los casos utilizara actualmente o hubiera utilizado en los últimos 12 meses la vía inyectada como forma de consumo de drogas. La combinación de estas dos condiciones permitió formar cuatro subgrupos con la siguiente distribución: 33 Usuarios de Drogas Inyectadas (UDIs) $\mathrm{VIH}+, 24$ UDIs VIH-, 17 No-UDIs VIH+ y 26 No-UDIs VIH-.

El análisis de las variables de caracterización ofrecen el siguiente perfil de participante en el estudio: Hombre (81\%), presenta una edad media de 33,62 años [Recorrido: 18-45; Desviación Estándar (DE): 4,6; el 39\% tienen edades entre los 30 y 35 años], principalmente solteros (57\%) y con convivencia en la familia de origen (49\%). Tienen escasa formación académica (un 72\% sólo alcanza estudios primarios) y están trabajando (33\%), en situación de paro (un $15 \%$ con subsidio, y un $5 \%$ sin el), o se hayan percibiendo algún tipo de prestación (29\%). Un 75\% de los usuarios refiere haber sido detenido en alguna ocasión, y en un 34\% de los casos han estado en prisión. La media de años desde que se iniciaron en el consumo de opiáceos es de 15,8 (DE: 5,1) años, con un rango intercuartil (RI) entre 11 y 19 años. La permanencia media en el programa de metadona es de 27,7 (DE: 22) meses (IR: 9-39); con una dosis media de 65 (DE: 23,1; IR: 40-75) mg/día. El 93\% de los sujetos han realizado algún tratamiento previo por su adicción a la heroína, siendo la modalidad más utilizada el dispositivo de salud ambulatorio (74\%), seguido de la unidad hospitalaria de desintoxicación (35\%) y de la comunidad terapéutica (46\%). Durante el último mes, un $27 \%$ de los casos ha consumido heroína y un $29 \%$ otras drogas (cocaína, otros estimulantes o alcohol). Respecto al estado orgánico, un 50\% de los participantes presentan marcadores serológicos de $\mathrm{VIH}$ y el $81 \%$ a hepatitis; un $14 \%$ han sido diagnosticado en alguna ocasión de tuberculosis, un 24\% han tenido una sobredosis alguna vez y un $28 \%$ han sido diagnosticados y tratados por otras patologías psiquiátricas. Atendiendo a las conductas de riesgo, un 57\% refieren haber utilizado en sus últimos consumos la vía inyectada, existiendo en un 7\% conductas de riesgo a este respecto (presta la jeringuilla y/o utiliza la usada por otro). Practican conductas sexuales de riesgo un 35\%, existiendo en conjunto una prevalencia de conductas de riesgo del $36 \%$.

\section{Procedimiento}

Todos los participantes fueron entrevistados por el mismo clínico quien estableció los diagnósticos a partir de los criterios del DSM-IV, y recogió los datos 
sociodemográficos y de historia de consumo basados en algunas dimensiones del OTI ${ }^{30,31}$ y del $\mathrm{ASI}^{32,33}$. En una segunda sesión, se aplicaron tres instrumentos de medida de calidad de vida: el IDUQoL, el SF-36 y el EQ-5D, estos dos últimos administrados una vez concluido el IDUQoL. Asimismo, se tomaron datos del registro de actividades de tratamiento tales como la dosis de metadona, la prevalencia de consumo de drogas (heroína y cocaína) a partir de los urinocontroles realizados según protocolo e incidencias de mal cumplimiento del programa.

Se seleccionaron al azar cincuenta de los participantes para la realización de un retest pasados entre diez y catorce días posteriores a la entrevista de valoración del IDUQoL. Dos de ellos rehusaron la entrevista de retest y fueron sustituidos por otros dos, también elegidos de forma aleatoria.

\section{Instrumentos}

IDUQoL .- Para el presente estudio se ha utilizado la versión revisada de 21 ítems propuesta en el estudio del grupo de Hubley ${ }^{22}$. Valora otros tantos dominios de la calidad de vida considerados como importantes entre los usuarios de drogas, a saber: 1) la salud, 2) el tratamiento por problemas de droga, 3) los cuidados de salud, 4) la reducción de daños, 5) el trato dispensado por otros, 6) el sentimiento o percepción de utilidad, 7) el sentimiento de bienestar con uno mismo (autoestima), 8) la independencia y libertad de opción, 9) la espiritualidad, 10) las drogas y el alcohol, 11) el dinero (recursos económicos), 12) el alojamiento o albergue (lugar de residencia), 13) el transporte, 14) la seguridad del vecindario, 15) los recursos comunitarios, 16) las actividades de ocio, 17) la educación, 18) las amistades, 19) la familia, 20) las relaciones de pareja, y 21) el sexo o relaciones sexuales. Los participantes son preguntados por el grado de satisfacción experimentada en cada uno de estos dominios a partir de una escala tipo Likert con valores que van desde 1 (muy insatisfecho) a 7 (muy satisfecho). Tanto la presentación de los dominios como la respuesta a los mismos se realizaba a partir de representaciones gráficas que servían como estímulos elicitadores. Los dominios se presentaban en unas tarjetas con la denominación correspondiente y un motivo gráfico, figurando en el anverso una explicación de lo que pretende valorar; para la valoración se les ofrecía una tarjeta donde aparecía una numeración del 1 a 7 y sobre cada número un emoticono que variaba desde una cara muy enojada - insatisfecha (valor 1) a una cara muy satisfecha (valor 7). Una vez habían respondido a todos los estímulos, se les ofrecía el conjunto de 21 tarjetas para que las ordenaran de mayor a menor importancia en relación a lo que ellos entendían por calidad de vida. Finalmente, se ha calculado una pun- tuación global del IDUQoL a partir de la suma promediada de los 21 dominios.

Cuestionario de Salud SF-3634,35.- Fue desarrollado para su uso en el Estudio de Resultados Médicos (Medical Outcome Study - MOS) a partir de una amplia batería de cuestionarios que incluía 40 conceptos relacionados con la salud. La adaptación del cuestionario para su uso en España ha sido realizada por el equipo de Alonso ${ }^{36,37}$, mostrando niveles adecuados de validez, fiabilidad y equivalencia cultural con otras versiones de otros países $^{38}$. Los ítems del cuestionario informan tanto de estados positivos como negativos de la salud física y del bienestar emocional, identificando ocho dimensiones de salud: Función Física $(F F)$, limitaciones de Rol por problemas Físicos $(R F)$, Dolor Corporal (D), Salud General (SG), Vitalidad (V), Función Social (FS), limitaciones de Rol por problemas Emocionales (RE) y Salud Mental (SM). Asimismo, a partir de la combinación de las puntuaciones de cada dimensión pueden ser calculados dos puntuaciones resumen: la suma del componente físico (PCS - Physical Component Summary) y la suma del componente mental (MCS - Mental Component Summary). Para cada dimensión del SF-36, los ítems se codifican, agregan y transforman en una escala con recorrido desde 0 (peor estado de salud) hasta 100 (mejor estado de salud) utilizando los algoritmos e indicaciones que ofrece el manual de puntuación e interpretación del cuestionario ${ }^{35,39}$. Por tanto, una mayor puntuación en las diferentes dimensiones indica un mejor estado de salud y/o una mejor calidad de vida.

EQ-5D40, 41 .- Es un cuestionario genérico de CVRS, que produce un valor índice para su uso en los estudios de coste-utilidad. Consta de un sistema descriptivo del estado de salud con 5 dimensiones de 3 ítems cada una, que definen 3 grados de gravedad, y una escala visual analógica (EVA) cuyos extremos van de 0 (peor estado de salud imaginable) a 100 (mejor estado de salud imaginable). La combinación de las respuestas a las 5 dimensiones permite obtener una 'tarifa' o valor índice que expresa el grado de gravedad atribuida a esa combinación determinada. En el presente estudio se calculó el valor índice utilizando el método propuesto por Prieto y Sacristán ${ }^{42}$.

\section{Estrategia de análisis}

El estudio de valoración psicométrica del IDUQoL queda constituido por las siguientes fases. En primer lugar se ha valorado la matriz de intercorrelaciones entre los 21 dominios componentes del instrumento (coeficientes de correlación de Sperman: $r_{s}$ ); y entre los distintos dominios y el índice global (en este caso 
se han estimado tanto $r_{s}$ como coeficientes de correlación semi-parcial: $r_{\text {sp }}$ ). Asimismo, por un lado, como medida de su consistencia interna se ha obtenido el coeficiente alpha de Cronbach $(\alpha)$ del total de la escala y el resultante si se eliminase cada uno de los ítems componentes; y, por otro lado, se han estimado los Coeficientes de Correlación Intraclase (CCI) para el análisis de las asociaciones test-retest como medida de su estabilidad. Ambas estimaciones forman parte del análisis de fiabilidad del instrumento.

Esta primera fase de análisis permitirá una aproximación a la valoración de la unidimensionalidad planteada para el instrumento por las autoras del IDUQoL ${ }^{22}$, que será completada mediante la utilización de técnicas de factorización, utilizándose en nuestro caso un primer análisis de tipo exploratorio a través de un análisis factorial de componentes principales, previa comprobación de las condiciones de aplicabilidad (pruebas de Kaiser-Meyer-Olkin y esfericidad de Bartlett) ${ }^{29,43}$. Asimismo, se ha contrastado el modelo unifactorial mediante la aplicación de un análisis factorial confirmatorio utilizando el programa de ecuaciones estructurales EQS ${ }^{44}$. Se ha empleado un método de máxima verosimilitud, utilizando los siguientes indicadores de bondad de ajuste: 1) Ji cuadrado del modelo de independencia; 2) Ji cuadrado dividido por los grados de libertad (el cociente debe ser menor de 4; a menor índice, mejor ajuste), 3) el índice de ajuste comparativo de Bentler (CFI, Comparative Fit Index), 4) el índice de bondad de ajuste LISREL (GFI, Goodness of Fit Index), 5) la raíz cuadrada media residual estandarizada (SRMR, Standardized Root Mean square Residual), y 6) la raíz cuadrada media de error de aproximación (RMSEA, Root Mean Square Error of Approximation). Para que exista un buen ajuste, los valores CFI y GFI deberían superar el valor 0,90 (cuanto mayor sea el valor, mejor es el ajuste), y los valores SRMR y RMSEA deberían ser menores o iguales a 0,05 (cuanto menor sea el valor, mejor ajuste) ${ }^{44-46}$. Los resultados de ambas estrategias de análisis factorial conformaran evidencia sobre la validez de constructo del IDUQoL.

En otro ámbito de análisis, también se ha tratado de valorar la convergencia del IDUQoL con otros constructos afines, para lo cual se han calculado los coeficientes de correlación de Spearman entre el Índice Global y los dominios del IDUQoL con los índices y dimensiones de dos instrumentos de calidad de vida ampliamente aceptados y utilizados en la investigación internacional: el cuestionario de salud SF-36 y el índice de utilidad EQ-5D. La convergencia y divergencia entre los dominios del IDUQoL y los ofrecidos por los dos instrumentos referidos mostrarán datos para el estudio de la validez concurrente ${ }^{47,48}$.

Por último, también se ha probado la validez de criterio y discriminante asociando el IDUQoL con varia- bles contextuales y de tratamiento con las cuales se esperan ciertos patrones de asociación. Se ha procedido para ello mediante dos estrategias ${ }^{49,50}$. Por un lado, se han analizado las correlaciones de los dominios e índice global del IDUQoL con un conjunto de variables criterio de forma similar al procedimiento seguido por Hubley². La segunda estrategia parte de un a priori conceptual. La IDUQoL se creó en el seno de una investigación más amplia sobre población afectada por VIH/SIDA y, como su nombre lo indica, es específica para personas que hacen uso de la vía inyectada para la administración de la droga. Entre los criterios de selección de la muestra se encontraban estas dos condiciones de modo que se comparará los resultados de la IDUQoL (índice global) entre los participantes inyectores y no inyectores, entre los $\mathrm{VIH}+$ y los VIH-, y entre los grupos de participantes que resulten de la combinación de ambos criterios. Para la primera estrategia se obtendrán los coeficientes de correlación de Spearman, y en la segunda se realizará un contraste de medias entre los grupos resultantes de la combinación de los criterios propuestos, realizándose un análisis de varianza utilizando la prueba robusta de Brown-Forsythe ${ }^{51}$.

En el apartado de resultados se expondrán comentarios sobre los análisis específicos realizados, con objeto de optimizar la comprensión de los datos obtenidos.

\section{RESULTADOS}

El IDUQoL, al igual que el resto de instrumentos, fue respondido por todos los participantes entrevistados, no produciéndose pérdida de respuestas.

\section{Fiabilidad}

El análisis de las interrelaciones entre los dominios del IDUQoL y los datos de fiabilidad del instrumento valorados a través del coeficiente alpha de Cronbach $(\alpha)$ y el Coeficiente de Correlación Intraclase (CCI) se presentan en la Tabla 1. La consistencia interna del instrumento ha mostrado un coeficiente de fiabilidad muy alto $(\alpha=0,92)$ y la retirada de ninguno de los dominios permite mejorar dicho valor. El coeficiente de correlación Intraclase del índice global del IDUQoL como medida de fiabilidad test-retest ha sido alto $(\mathrm{CCl}=0,79)$, variando en el caso de las dimensiones entre valores de 0,05 a 0,72. En dos dominios del IDUQoL el coeficiente logrado no ha mostrado significación estadística; el caso de la reducción de daños $(\mathrm{CCl}=0,05)$ y el transporte $(\mathrm{CCl}=0,08)$. 


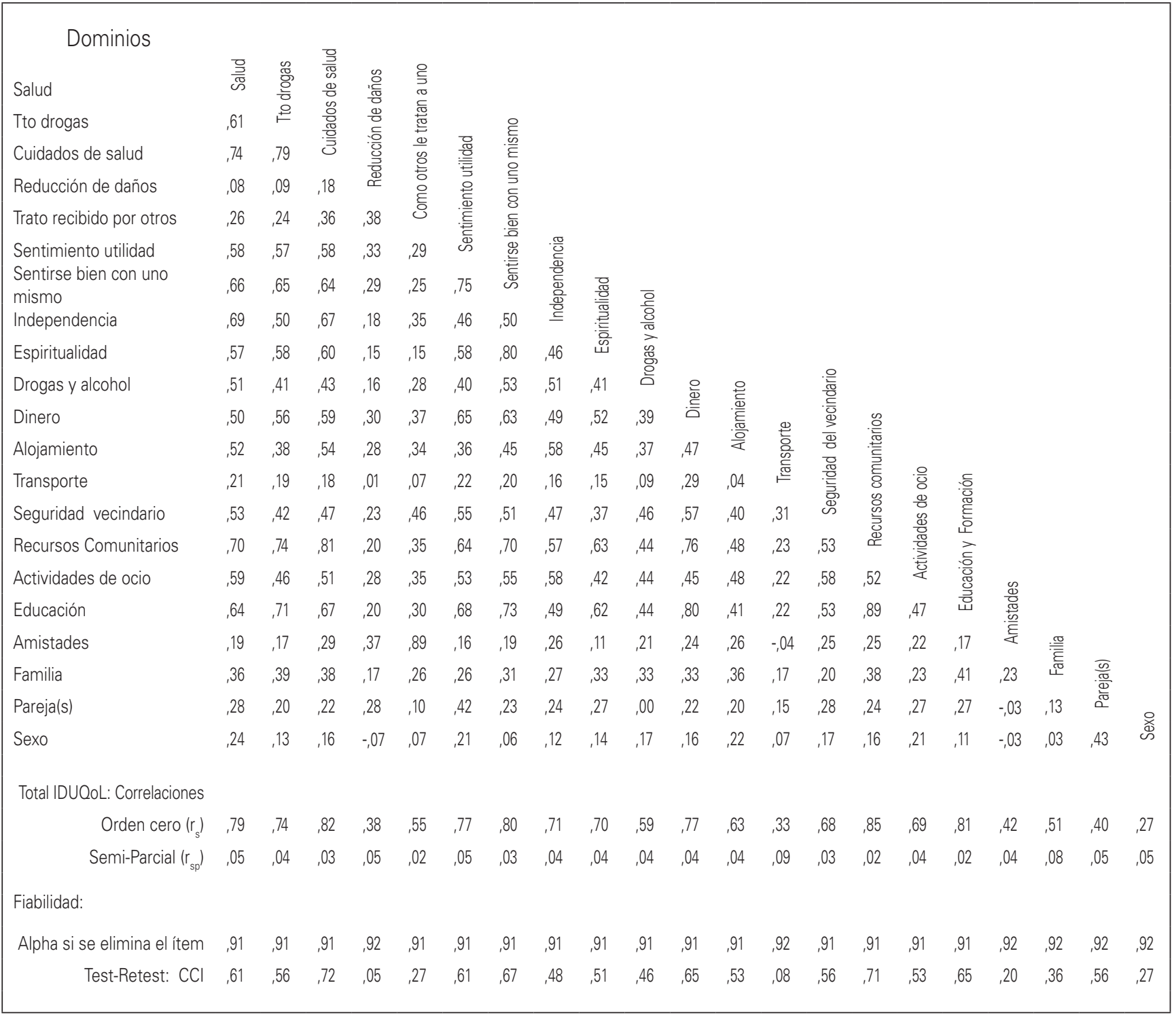

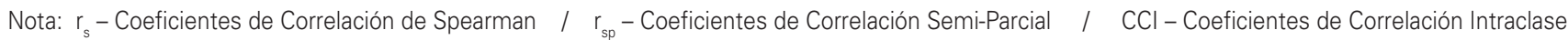
Puntuaciones iguales o superiores a 0,18 presentan significación estadística $(p<0,05)$

De las 210 correlaciones inter-dominios, salvo en cuatro casos, todas han resultado de signo positivo y en 169 asociaciones han mostrado significación estadística. Los coeficientes han variado entre un valor mínimo de 0,00 (asociación entre drogas y pareja) a uno máximo de 0,89 (asociación entre amistades y el trato dispensado por otros), con una correlación media de 0,37 descrita por Clark y Watson ${ }^{47}$ como aceptable. Los dominios de 'Sexo', 'Transporte' y 'Reducción de daños' serían los que presentan un menor número de asociaciones significativas con otros dominios (5, 9 y 11 respectivamente sobre un total de 20 posibles asociaciones).
Por otro lado, las asociaciones de los 21 dominios con el índice global del IDUQoL presentan en todos los casos significación estadística con coeficientes que oscilan entre 0,27 y 0,85. En 10 de los 21 casos el coeficiente de correlación logrado es superior al valor de 0,70 , considerado como muy adecuado ${ }^{47}$. También se han calculado los coeficientes de correlación semiparcial $\left(r_{\text {sp }}\right)$, los cuales nos ofrecen información sobre la contribución particular de cada uno de los dominios una vez se ha controlado la variabilidad común explicada por el conjunto de los mismos. En nuestro caso, nos interesa encontrar coeficientes bajos lo cual indicará que el conjunto de los ítems magnifican su variabilidad conjunta y no ofrecen un patrón particular. En todos los casos los coeficientes semiparciales 


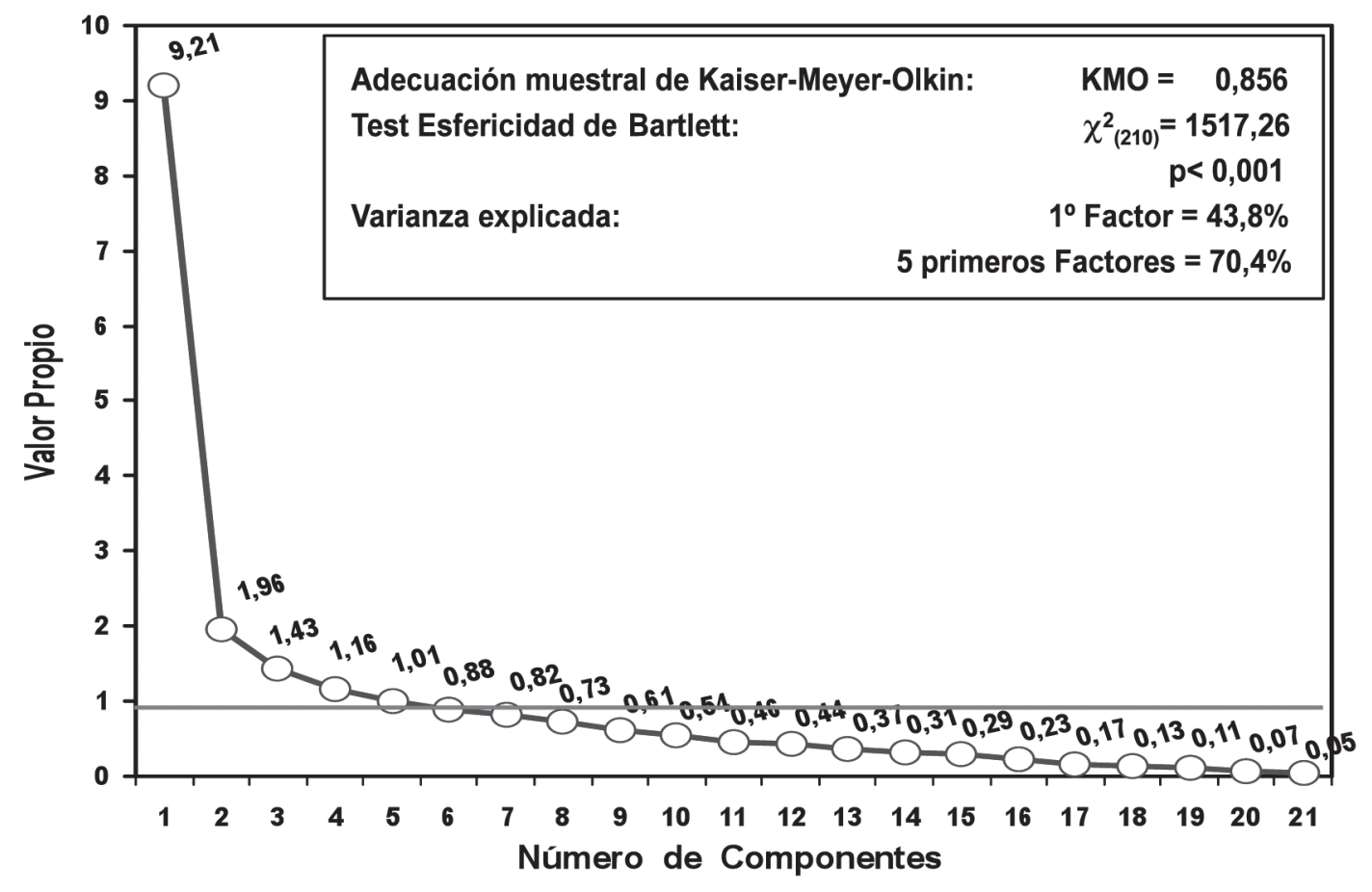

Figura 1. Análisis Factorial Exploratorio del IDUQoL: Gráfico de segmentación y pruebas de adecuación

encontrados no superan el valor 0,10 y en su mayoría oscilan entre los valores de 0,02 y 0,05. Se apartan un poco de esta tónica los dominios de 'transporte' $\left(r_{\text {sp }}=0,09\right)$ y 'familia' $\left(r_{\text {sp }}=0,08\right)$, pero en general estos datos apuntan hacia la valoración de una adecuada consistencia inter-dominios y sería una primera prueba de su unidimensionalidad 27, 28,48.

\section{Análisis Factorial Exploratorio (AFE) y Confirmato- rio (AFC)}

En párrafos anteriores hemos utilizado un índice global de la IDUQoL pero ello sólo es factible si el instrumento muestra unidimensionalidad, es decir, el conjunto de dominios se subsumen en un factor común más simple que agrupa su diversidad. Para poder factorizar el conjunto de dominios es necesario comprobar la adecuación de la matriz de correlaciones mediante las pruebas de esfericidad de Bartlett y el criterio de adecuación muestral de Kaiseer-MeyerOlkin (Figura 1) que han resultado óptimos. El proceso de factorización concluye con 8 iteracciones y la obtención de 5 factores con valores propios (eigenvalue) superiores a 1. El primer factor obtiene un autovalor de 9,21 y explica un 43,8\% de la varianza. La ratio entre el primer factor y el segundo es de 4,69 $(9,21 / 1,96)$ superando el criterio propuesto por Hattie $^{27,28}$ y Gorsuch ${ }^{29}$ [valor crítico > 4] como evidencia de unidimensionalidad.
Este primer resultado nos permite considerar la unidimensionalidad de la escala IDUQoL y, por tanto, la factibilidad de optar por una medida global. No obstante, creemos oportuno analizar el resto del análisis factorial. Dada la alta inter-correlación entre los dominios y la extracción de un factor dominante, se solicitó una rotación oblicua (no ortogonal) para valorar la saturación de los pesos factoriales en los cinco componentes con valor propio superior a 1. En la Tabla 2 se presentan los datos de las comunalidades y los pesos factoriales distribuidos entre los factores resultantes. En el caso del primer factor se presentan el total de pesos, mientras que en los cuatro restantes sólo se consideran aquellos mayores de 0,35. El proceso de rotación, basado en una normalización Oblimin con Kaiser, converge en 15 interacciones, haciendo un nuevo reparto de la varianza explicada, de modo que el primer factor explicaría un 31,8\%, el segundo y el tercero un $12 \%$ cada uno, y un $8,3 \%$ y $6,3 \%$ el cuarto y quinto factor respectivamente. Salvo el caso del dominio de Familia (comunalidad $=0,27$ ), el resto presentan comunalidades por encima del valor 0,50; es decir, existe un alto grado de varianza común entre los dominios. Trece dominios saturan con pesos entre 0,36 y 0,92 en el primer factor. El segundo factor agrupa los dominios de Amistades $(0,95)$, Trato dispensado por otros $(0,95)$ y con menor carga el de Reducción de daños $(0,52)$. En el tercer factor saturan el Sexo $(0,86)$ y la Pareja $(0,73)$; en el cuarto la Pareja $(0,46)$, la Reducción de daños $(0,64)$ y las Drogas 
Tabla 2

Análisis factorial de componentes principales de la IDUQoL

\begin{tabular}{|c|c|c|c|c|c|c|c|}
\hline & & \multirow[b]{2}{*}{ Comunalidad } & \multicolumn{5}{|c|}{ Saturaciones Factoriales } \\
\hline & & & $\mathbf{F 1}$ & $\mathbf{F 2}$ & F3 & F4 & F5 \\
\hline IDUFG & Sentirse bien con uno mismo & 0,79 & 0,92 & & & & \\
\hline IDUED & Educación & 0,82 & 0,90 & & & & \\
\hline IDUSP & Espiritualidad & 0,68 & 0,88 & & & & \\
\hline IDURC & Recursos comunitarios & 0,82 & 0,87 & & & & \\
\hline IDUDT & Tto drogas & 0,69 & 0,86 & & & & \\
\hline IDUHC & Cuidados de salud & 0,76 & 0,79 & & & & \\
\hline IDUBU & Sentimiento de utilidad & 0,74 & 0,69 & & & & \\
\hline IDUMO & Dinero & 0,70 & 0,69 & & & & \\
\hline IDUHE & Salud & 0,76 & 0,67 & & & & \\
\hline IDUIN & Independencia & 0,66 & 0,50 & & & & \\
\hline IDUDR & Drogas & 0,51 & 0,45 & & & $-0,36$ & \\
\hline IDUFA & Familia & 0,27 & 0,45 & & & & \\
\hline IDUHO & Alojamiento & 0,59 & 0,43 & & & & \\
\hline IDULA & Actividades de ocio & 0,58 & 0,36 & & & & \\
\hline IDUFR & Amistades & 0,85 & $-0,07$ & 0,95 & & & \\
\hline IDUTO & Trato dispensado por otros & 0,88 & $-0,07$ & 0,95 & & & \\
\hline IDUSX & Sexo & 0,70 & $-0,14$ & & 0,86 & & \\
\hline IDUPA & Pareja & 0,79 & 0,08 & & 0,73 & 0,46 & \\
\hline IDUHR & Reducción de daños & 0,72 & 0,12 & 0,52 & & 0,64 & \\
\hline IDUTR & Transporte & 0,80 & $-0,03$ & & & & 0,90 \\
\hline \multirow[t]{3}{*}{ IDUNS } & Seguridad del vecindario & 0,66 & 0,27 & & & & 0,44 \\
\hline & & Valor propio & 9,21 & 1,96 & 1,42 & 1,15 & 1,03 \\
\hline & Varianza Explicada tras & otación ( 70,4\%) & 31,8 & 12,0 & 11,9 & 8,3 & 6,3 \\
\hline
\end{tabular}

Nota.- Comunalidad: Expresa la parte de cada variable que puede ser explicada por los factores comunes a ella F1 a F5 .- Cada una de las dimensiones (factores) resultantes de la factorización

$(-0,36)$; y en el quinto el Transporte $(0,90)$ y la Seguridad del vecindario $(0,44)$. Podemos apreciar que algunos de estos factores presentan una agrupación lógica, de sentido común, tal es el caso del segundo, tercer y quinto factor, y en otros resulta un tanto confusa (el factor cuarto y el papel del dominio Reducción de daños en el segundo factor).

Por otro lado, se ha realizado un análisis factorial confirmatorio proponiendo la unidimensionalidad de la escala, obteniéndose índices de bondad de ajuste muy adecuados $\left(\chi_{(189)}^{2}=225,95 ; p=0,034 ; \chi^{2} / g l=\right.$ 1,19; $\mathrm{GFI}=0,92 ; \mathrm{CFI}=0,97 ; \mathrm{SRMR}=0,046 ; \mathrm{RMSEA}=$ $0,044)$ con pesos factoriales entre 0,66 y 0,78. Estos resultados, junto a los del factorial exploratorio, el análisis de la matriz de correlaciones y de las correlaciones semiparciales de los dominios respecto al índice global, presentan base suficiente para admitir la unidimensionalidad del IDUQoL.

\section{Validez concurrente}

En la Tabla 3 se presenta la matriz de correlaciones que analiza la convergencia del IDUQoL con otros constructos afines valorados a través del SF-36 y el EQ-5D. El índice global del IDUOoL muestra asociación positiva y con significación estadística con todas las dimensiones e índices de los instrumentos en cuestión: Coeficientes entre 0,32 y 0,59 con las dimensiones e índices sumarios del SF-36; y una correlación de 0,61 y 0,58 con la escala visual analógica e índice compuesto del EQ-5D, respectivamente. Ello índica que el grado de calidad de vida valorado con el IDUQoL se asocia al grado de calidad de vida o estado de salud valorados con el SF-36 y EQ-5D. Asimismo, los diferentes dominios del IDUQoL se asocian con las variables criterio con el mismo patrón que lo ha hecho el índice global, aunque con valores de asociación quizá un poco más ajustados en la mayoría de los casos. Las variables criterio consideradas 
Tabla 3

Matriz de correlaciones entre los dominios del IDUQoL y las dimensiones e índices Sumarios del SF-36 y del EQ-5D (Validez convergente)

\begin{tabular}{|c|c|c|c|c|c|c|c|c|c|c|c|c|}
\hline & \multicolumn{10}{|c|}{ SF-36 } & \multicolumn{2}{|c|}{ EQ-5D } \\
\hline & \multicolumn{8}{|c|}{ Dimensiones SF } & \multicolumn{2}{|c|}{ Sumarios SF } & & \\
\hline & PF & $\mathrm{RP}$ & $\mathrm{BP}$ & $\mathrm{GH}$ & VT & SF & $\mathrm{RE}$ & $\mathrm{MH}$ & PCS & MCS & EVA & Índice \\
\hline Índice Global IDUQoL & 0,40 & 0,47 & 0,43 & 0,55 & 0,57 & 0,40 & 0,32 & 0,59 & 0,47 & 0,43 & 0,61 & 0,58 \\
\hline Salud & 0,50 & 0,44 & 0,41 & 0,68 & 0,57 & 0,43 & 0,38 & 0,53 & 0,51 & 0,44 & 0,49 & 0,54 \\
\hline Tratamiento Drogas & 0,22 & 0,37 & 0,41 & 0,33 & 0,51 & 0,43 & 0,18 & 0,42 & 0,37 & 0,34 & 0,40 & 0,48 \\
\hline Cuidados de Salud & 0,39 & 0,48 & 0,45 & 0,50 & 0,54 & 0,37 & 0,28 & 0,46 & 0,50 & 0,35 & 0,45 & 0,56 \\
\hline Reducción de daños & 0,01 & $-0,02$ & 0,07 & 0,07 & 0,10 & 0,05 & 0,07 & 0,28 & $-0,05$ & 0,17 & 0,36 & 0,16 \\
\hline Trato recibido por otros & 0,25 & 0,27 & 0,12 & 0,23 & 0,22 & 0,07 & 0,02 & 0,22 & 0,27 & 0,08 & 0,37 & 0,21 \\
\hline Sentimiento de utilidad & 0,30 & 0,24 & 0,36 & 0,40 & 0,46 & 0,42 & 0,35 & 0,64 & 0,22 & 0,51 & 0,61 & 0,51 \\
\hline Sentirse bien con uno mismo & 0,34 & 0,37 & 0,40 & 0,41 & 0,49 & 0,43 & 0,45 & 0,59 & 0,28 & 0,52 & 0,59 & 0,53 \\
\hline Independencia & 0,47 & 0,34 & 0,43 & 0,54 & 0,43 & 0,27 & 0,24 & 0,33 & 0,51 & 0,24 & 0,40 & 0,42 \\
\hline Espiritualidad & 0,32 & 0,38 & 0,36 & 0,37 & 0,46 & 0,38 & 0,38 & 0,48 & 0,31 & 0,43 & 0,42 & 0,53 \\
\hline Drogas & 0,21 & 0,23 & 0,17 & 0,32 & 0,18 & 0,16 & 0,15 & 0,27 & 0,23 & 0,18 & 0,31 & 0,26 \\
\hline Dinero & 0,17 & 0,42 & 0,30 & 0,36 & 0,36 & 0,29 & 0,24 & 0,41 & 0,32 & 0,31 & 0,53 & 0,39 \\
\hline Alojamiento & 0,24 & 0,36 & 0,21 & 0,38 & 0,31 & 0,19 & 0,23 & 0,39 & 0,29 & 0,27 & 0,38 & 0,37 \\
\hline Transporte & 0,03 & 0,15 & 0,10 & 0,12 & 0,12 & 0,13 & 0,13 & 0,22 & 0,06 & 0,17 & 0,18 & 0,11 \\
\hline Seguridad del vecindario & 0,25 & 0,29 & 0,20 & 0,42 & 0,36 & 0,29 & 0,16 & 0,35 & 0,31 & 0,26 & 0,47 & 0,30 \\
\hline Recursos comunitarios & 0,29 & 0,52 & 0,45 & 0,40 & 0,44 & 0,36 & 0,23 & 0,39 & 0,49 & 0,29 & 0,48 & 0,53 \\
\hline Actividades de ocio & 0,37 & 0,35 & 0,47 & 0,40 & 0,46 & 0,25 & 0,29 & 0,44 & 0,40 & 0,32 & 0,45 & 0,42 \\
\hline Educación & 0,23 & 0,42 & 0,34 & 0,32 & 0,42 & 0,35 & 0,26 & 0,41 & 0,33 & 0,34 & 0,46 & 0,48 \\
\hline Amistades & 0,24 & 0,19 & 0,07 & 0,20 & 0,10 & 0,00 & $-0,02$ & 0,18 & 0,22 & 0,01 & 0,23 & 0,16 \\
\hline Familia & 0,24 & 0,20 & 0,20 & 0,32 & 0,31 & 0,09 & 0,01 & 0,24 & 0,30 & 0,10 & 0,21 & 0,35 \\
\hline Pareja & 0,06 & 0,07 & 0,12 & 0,27 & 0,45 & 0,24 & 0,17 & 0,30 & 0,08 & 0,31 & 0,26 & 0,21 \\
\hline Sexo & 0,11 & 0,14 & 0,12 & 0,30 & 0,28 & 0,17 & 0,07 & 0,18 & 0,20 & 0,14 & 0,16 & 0,10 \\
\hline
\end{tabular}

Nota.- Correlaciones superiores a 0,18 son estadísticamente significativas para $p<0,05$

SF-36: PF - Funcionamiento Físico; RP - Limitación de Rol por problemas físicos; BP - Dolor Corporal; GH - Salud General; VT - Vitalidad; SF - Funcionamiento Social; RE - Limitación de Rol por problemas emocionales; MH - Salud mental; PCS - Índice Sumario de Salud Física; MCS - Índice Sumario de Salud Mental.

EQ-5D - EuroQoL: EVA - Escala Visual Analógica; Índice - Índice Compuesto Global.

valoran de forma más específica la calidad de vida relacionada con la salud, y precisamente los dominios del IDUQoL que tienen que ver con la salud (Salud, Tratamiento por Drogas y Cuidados de salud) o con el estado emocional (Sentimiento de utilidad, Sentirse bien con uno mismo, Independencia y Espiritualidad) son los que muestran coeficientes de correlación más elevados. Por su parte, dimensiones como el Transporte, las Amistades, la Pareja o el Sexo, apenas muestran asociaciones estadísticamente significativas y en el caso de que aparezcan lo son de baja magnitud. Un dato que nos ha sorprendido es el bajo grado de asociación del dominio de Reducción de daños respecto al SF-36 y EQ-5D. Una posible explicación radique en que los sujetos participantes se hallan en tratamiento con metadona y, por ello, utilicen menos los recursos destinados a la reducción de daños (intercambio de jeringuillas, salas de consumo higiénico, etc.) que otros que se hallan en un consumo activo o sin tratamiento como era el caso del estudio canadiense. En definitiva, y en general, respecto a la validez concurrente del IDUQoL, podemos decir que los datos observados refrendan tanto un adecuado valor de convergencia como de validez divergente. 
Tabla 4

Matriz de correlaciones entre los dominios del IDUQoL y medidas criterio (validez concurrente)

\begin{tabular}{|c|c|c|c|c|c|c|c|c|c|c|c|c|c|c|}
\hline & 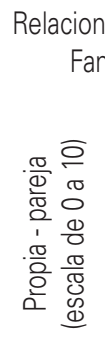 & 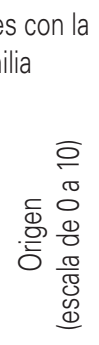 & 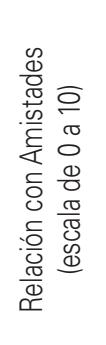 & 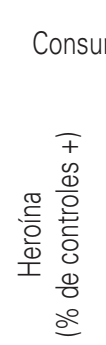 & 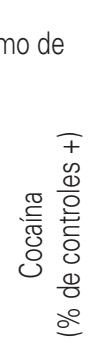 & 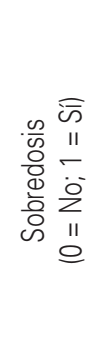 & 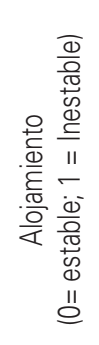 & 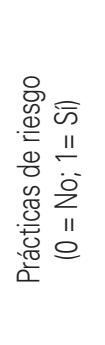 & 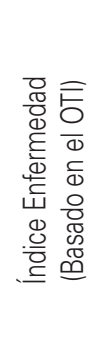 & 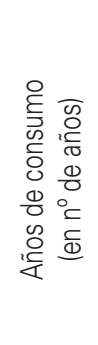 & $\begin{array}{l}\overline{0} \\
\text { 요 }\end{array}$ & 离 & 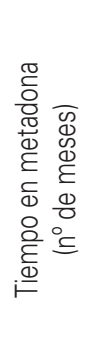 & 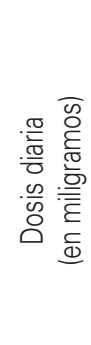 \\
\hline IDUQOL & 0,25 & 0,19 & 0,31 & $-0,03$ & $-0,05$ & $-0,15$ & $-0,15$ & $-0,26$ & $-0,32$ & $-0,10$ & $-0,13$ & $-0,01$ & $-0,03$ & $-0,21$ \\
\hline IDUHE & 0,14 & 0,11 & 0,10 & $-0,04$ & $-0,09$ & $-0,20$ & $-0,22$ & 0,01 & $-0,41$ & $-0,10$ & $-0,14$ & 0,11 & $-0,09$ & $-0,21$ \\
\hline IDUDT & 0,21 & 0,09 & 0,08 & $-0,11$ & $-0,15$ & $-0,11$ & $-0,27$ & $-0,07$ & $-0,22$ & 0,02 & 0,00 & 0,01 & 0,07 & $-0,12$ \\
\hline IDUHC & 0,22 & 0,09 & 0,12 & $-0,05$ & $-0,11$ & $-0,16$ & $-0,23$ & $-0,08$ & $-0,32$ & $-0,08$ & $-0,12$ & 0,01 & $-0,09$ & $-0,19$ \\
\hline IDUHR & 0,27 & 0,09 & 0,32 & 0,04 & 0,11 & $-0,09$ & 0,06 & $-0,77$ & $-0,17$ & 0,03 & 0,01 & 0,04 & $-0,06$ & 0,00 \\
\hline IDUTO & 0,12 & 0,15 & 0,60 & 0,03 & $-0,19$ & $-0,16$ & 0,09 & $-0,16$ & $-0,08$ & $-0,12$ & $-0,12$ & $-0,08$ & $-0,02$ & $-0,07$ \\
\hline IDUBU & 0,15 & 0,01 & 0,22 & $-0,14$ & 0,01 & $-0,02$ & $-0,17$ & $-0,35$ & $-0,26$ & 0,02 & $-0,01$ & $-0,07$ & 0,04 & $-0,12$ \\
\hline IDUFG & 0,13 & 0,08 & 0,15 & $-0,03$ & $-0,01$ & 0,00 & $-0,12$ & $-0,25$ & $-0,25$ & $-0,05$ & $-0,09$ & 0,07 & $-0,01$ & $-0,15$ \\
\hline IDUIN & 0,20 & 0,09 & 0,20 & 0,06 & $-0,13$ & $-0,17$ & $-0,09$ & $-0,04$ & $-0,29$ & $-0,10$ & $-0,09$ & 0,05 & $-0,00$ & $-0,07$ \\
\hline IDUSP & 0,12 & 0,09 & 0,02 & 0,01 & $-0,04$ & $-0,06$ & $-0,07$ & $-0,14$ & $-0,29$ & $-0,03$ & $-0,08$ & 0,12 & 0,01 & $-0,21$ \\
\hline IDUDR & 0,08 & 0,19 & 0,10 & $-0,27$ & $-0,11$ & $-0,18$ & $-0,09$ & $-0,05$ & $-0,12$ & $-0,01$ & $-0,02$ & 0,10 & 0,02 & $-0,02$ \\
\hline IDUMO & $-0,06$ & $-0,04$ & 0,21 & $-0,09$ & 0,06 & $-0,13$ & $-0,15$ & $-0,20$ & $-0,13$ & $-0,11$ & $-0,19$ & $-0,09$ & $-0,09$ & $-0,19$ \\
\hline IDUHO & 0,21 & 0,19 & 0,19 & 0,06 & 0,02 & $-0,16$ & $-0,37$ & $-0,15$ & $-0,38$ & $-0,19$ & $-0,11$ & 0,06 & $-0,05$ & $-0,16$ \\
\hline IDUTR & 0,03 & 0,05 & 0,02 & $-0,14$ & 0,18 & 0,15 & $-0,05$ & $-0,13$ & 0,13 & 0,14 & 0,09 & 0,06 & 0,08 & $-0,05$ \\
\hline IDUNS & $-0,02$ & 0,06 & 0,30 & 0,02 & 0,07 & $-0,06$ & $-0,07$ & $-0,09$ & $-0,15$ & $-0,02$ & $-0,01$ & 0,00 & $-0,01$ & $-0,11$ \\
\hline IDURC & 0,18 & 0,02 & 0,17 & $-0,07$ & $-0,05$ & $-0,14$ & $-0,19$ & $-0,15$ & $-0,28$ & $-0,10$ & $-0,19$ & $-0,10$ & $-0,12$ & $-0,13$ \\
\hline IDULA & 0,17 & 0,09 & 0,19 & $-0,08$ & 0,07 & 0,04 & 0,02 & $-0,21$ & $-0,23$ & $-0,07$ & $-0,04$ & $-0,01$ & 0,08 & $-0,17$ \\
\hline IDUED & 0,13 & 0,06 & 0,10 & $-0,09$ & $-0,01$ & $-0,13$ & $-0,21$ & $-0,19$ & $-0,21$ & $-0,13$ & $-0,18$ & $-0,16$ & $-0,10$ & $-0,17$ \\
\hline IDUFR & 0,09 & 0,14 & 0,68 & 0,05 & $-0,24$ & $-0,16$ & 0,13 & $-0,16$ & $-0,15$ & $-0,11$ & $-0,12$ & $-0,01$ & $-0,03$ & $-0,03$ \\
\hline IDUFA & 0,38 & 0,79 & 0,20 & 0,06 & $-0,06$ & $-0,11$ & $-0,07$ & $-0,19$ & $-0,08$ & $-0,09$ & $-0,16$ & $-0,05$ & 0,04 & $-0,18$ \\
\hline IDUPA & 0,38 & 0,07 & $-0,01$ & $-0,04$ & 0,04 & $-0,09$ & $-0,09$ & $-0,21$ & $-0,21$ & $-0,10$ & $-0,06$ & $-0,10$ & $-0,06$ & $-0,23$ \\
\hline IDUSX & 0,24 & $-0,02$ & $-0,05$ & 0,00 & 0,06 & $-0,12$ & $-0,08$ & 0,16 & $-0,18$ & $-0,13$ & $-0,02$ & $-0,18$ & 0,02 & $-0,23$ \\
\hline
\end{tabular}

Nota.- Correlaciones superiores a 0,18 son estadísticamente significativas para $p<0,05$. Se resaltan en cursiva y negrita las que muestran significación estadísticamente significativa y asociación teórica

IDUQoL - Índice Global; IDUHE - Salud; IDUDT - Tto drogas; IDUHC - Cuidados de salud; IDUHR - Reducción de daños; IDUTO - Trato recibido por otros; IDUBU - Sentimiento de utilidad; IDUFG - Sentirse bien uno mismo; IDUIN - Independencia; IDUSP - Espiritualidad; IDUDR - Drogas; IDUMO - Dinero; IDUHO - Alojamiento; IDUTR - Transporte; IDUNS - Seguridad del vecindario; IDURC - Recursos comunitarios; IDULA - Actividades de ocio; IDUED - Educación; IDUFR - Amistades; IDUFA - Familia; IDUPA - Pareja; IDUSX Sexo. 


\section{Validez de criterio}

La asociación de la IDUQoL con otras variables criterio (Tabla 4) ofrece resultados en la línea de lo esperado. Así, por ejemplo, los dominios de Familia, Pareja y Sexo se asocian con la variable Relaciones con la familia propia ( $r=0,38 ; 0,38$ y 0,24 respectivamente), el dominio de Familia con Relaciones con la familia de origen $(r=0,79)$ y el dominio de Amistades con Relación con amistades $(r=0,68)$. Asimismo, una buena relación con los amigos se asocia positivamente a los dominios de Trato recibido por otros $(r=0,60)$, Seguridad del vecindario $(r=0,30)$, Dinero $(r=0,21)$ y Alojamiento $(r=0,19)$. El dominio de Drogas se asocia negativamente con una mayor frecuencia de consumo de heroína $(r=-0,27)$, aunque no de cocaína ( $r=$ $-0,11)$. Por su parte, una mejor salud valorada por el IDUQoL se asocia a una menor ocurrencia de sobredosis $(r=-0,20)$, a un alojamiento estable $(r=-0,22)$, a valores bajos en el índice de salud del OTI $(r=-0,41)$ y a una menor dosis de metadona $(r=-0,21)$. Tener practicas de riesgo se asocia a una peor valoración de la satisfacción con la Reducción de daños $(r=-0,77)$. Por su parte, también se aprecian correlaciones no significativas entre asociaciones de variables que no tienen por que estar relacionadas. En general, la matriz de correlaciones hallada muestra validez de criterio.

Atendiendo a la segunda estrategia propuesta para el análisis de la validez de criterio, se han obtenido los datos que se presentan en la Tabla 5. Observamos que ninguna de las variables consideradas (la vía de consumo, seropositividad a VIH, o la combinación de ambas) han mostrado un efecto estadísticamente significativo. Sí se aprecia una más baja puntuación en el IDUQoL entre los inyectores frente a los que no lo son, entre los $\mathrm{VIH}+$ frente a los $\mathrm{VIH}-$, y entre los $\mathrm{VIH}+$ inyectores frente al resto, pero en ninguno de los casos se ha producido significación estadística en la prueba de contraste de medias. Tan solo se aprecia un valor de probabilidad tendencial $(p=0,065)$ cuando se contempla la variable de seropositividad a VIH. En definitiva, estos resultados sugieren que el IDUQoL no discrimina de forma diferencial entre personas que utilizan distintas vías de consumo, lo que contradice el objetivo inicial propuesto por sus autoras y que da nombre al instrumento (injection drug user), pero sugiere que puede ser un buen instrumento de calidad de vida específico para el total de consumidores de drogas.

\section{DISCUSIÓN}

EI IDUQoL fue creado como un instrumento específico y sensible para la valoración de la calidad de vida percibida por usuarios de drogas inyectadas. Nuestro estudio ha mostrado los resultados de sus características psicométricas cuando es aplicado en una muestra española ofreciendo datos que lo ubican como un buen instrumento de medida.

Se han encontrado resultados muy similares a los obtenidos en los estudios de validación inicial con población drogodependiente del Canadá20-26 y a un reciente estudio de adaptación realizado por el grupo de Morales-Manrique ${ }^{52}$. En todos los estudios realizados se confirma la unidimensionalidad de la escala a través de múltiples procedimientos indicando una muy adecuada validez de constructo y la idoneidad

Tabla 5

Contraste de medias en la puntuación global del IDUQoL en función de la seropositividad a VIH y la vía de consumo

\begin{tabular}{|c|c|c|c|c|c|c|}
\hline & & $n$ & Media & $\mathrm{DE}$ & $\begin{array}{l}\text { Prueba de } \\
\text { Contraste }\end{array}$ & $\mathrm{p}$ \\
\hline & Total & 100 & 50,30 & 7,75 & & \\
\hline \multirow{2}{*}{ Uso de la Vía inyectada } & Inyector & 57 & 49,44 & 8,37 & 1,63 & 0,204 \\
\hline & No Inyector & 43 & 51,43 & 8,37 & & \\
\hline \multirow{2}{*}{ Seropositividad a VIH } & Positivo & 50 & 48,87 & 7,80 & 3,47 & 0,065 \\
\hline & Negativo & 50 & 51,73 & 7,51 & & \\
\hline \multirow{4}{*}{ Combinación de los dos criterios } & $\mathrm{VIH}+$ Inyector & 33 & 48,10 & 6,62 & 1,52 & 0,213 \\
\hline & $\mathrm{VIH}+\mathrm{No}$ Inyector & 17 & 50,37 & 9,74 & & \\
\hline & VIH- Inyector & 24 & 51,28 & 7,69 & & \\
\hline & VIH- No Inyector & 26 & 52,13 & 7,47 & & \\
\hline
\end{tabular}

Prueba de contraste: Análisis de varianza, Prueba robusta de Brown-Forsythe / p: valor de probabilidad 
para obtener un indicador sumario del instrumento. Su fiabilidad también resulta adecuada, con una alta consistencia interna y una apropiada reproductibilidad. Asimismo, presenta índices ajustados de convergencia con otros constructos afines de calidad de vida y una aceptable validez de criterio respecto a variables contextuales asociadas. Tomados de modo independiente, sus 21 dominios dan buena cuenta del repertorio de áreas de relevancia para la calidad de vida percibida por la personas con adicción a drogas, ofreciendo la posibilidad de tomar medidas específicas por dimensiones en caso de estudios más pormenorizados o delimitados $^{22}$. Esta convergencia de resultados en diferentes contextos culturales (Canadá vs España) y en distintos ámbitos geográficos dentro de una misma cultura (Valencia vs País Vasco), permite plantear la reproductibilidad del constructo valorado por el IDUQoL, lo cual constituiría una prueba más de la validez de contenido y de constructo del instrumento ${ }^{48}$.

No obstante, el IDUQoL presenta resultados que limitan su capacidad discriminante, al menos para satisfacer la especificidad del grupo para el que fue creada $^{20,21}$, el de inyectores de drogas, pero alternativamente sí podría ser planteada como una escala específica para el colectivo de consumidores de drogas, pudiéndose denominar como 'escala de calidad de vida para usuarios de drogas' tal y como plantea el grupo de Morales-Manrique ${ }^{52}$.

Por otro lado, la presentación del IDUQoL a través de estímulos gráficos para su cumplimentación, si bien puede requerir algo más de tiempo que los cuestionarios de 'lápiz y papel' auto o heteroaplicados, permite una respuesta más intuitiva, sobre todo entre aquellos con bajo nivel de estudios, y una mayor interacción y aproximación a la conceptualización de la calidad de vida que cada usuario de drogas tiene al respecto. Si bien el TECVASP ${ }^{18,19}$ es otro de los instrumentos específicos de calidad de vida desarrollado para población drogodependiente, éste hace un especial énfasis en las dimensiones asociadas al área de la salud, mientras el IDUQoL adopta un valoración más amplia y diversa del conjunto de espacios en el que se manifiesta la calidad de vida de los usuarios de drogas.

Si bien los resultados mostrados presentan la IDUQoL como un instrumento psicométricamente adecuado, han de contemplarse las limitaciones inherentes en el presente estudio y valorar los resultados como provisionales. Quizá la limitación más importante se halla en el tamaño de la muestra $(n=100)$, siendo conveniente nuevos estudios que permitan incrementar el tamaño de efectivos. No obstante, la convergencia de resultados del presente estudio con los obtenidos en el realizado en Valencia $(n=169)^{52}$, son prueba de su consistencia y reproductibilidad. Asimismo, sería conveniente diversificar, por un lado, las tipologías de sujetos participantes en función de la droga de consumo, y por otro, el uso del IDUQoL en momentos temporales pre-post como variable de resultado, en aras a evaluar de forma más efectiva, respectivamente, la validez discriminante y sensibilidad al cambio del instrumento.

En definitiva, los datos de la adaptación del IDUQoL a población española lo presentan como: 1) un instrumento específico de calidad de vida que recoge suficientemente las dimensiones implícitas en dicho constructo desde la perspectiva de los usuarios de drogas, 2) resulta fácil para ser comprendido y valorado, y 3) presenta un funcionamiento psicométrico altamente adecuado. Estas características confieren al IDUQoL un potencial importante para ser utilizado en los contextos clínicos y de investigación donde se contemple la calidad de vida como variable de resultado. A este respecto, se hacen necesarios nuevos estudios que valoren el comportamiento del IDUQoL cuando es utilizado para valorar el impacto de determinadas intervenciones, clínicas o psicosociales, respecto a su capacidad para estimar y discriminar las respuesta de cambio.

\section{REFERENCIAS}

1. Hilton BA, Thompson R, Moore-Dempsey L, Janzen $R G$. Harm reduction theories and strategies for control of human immunodeficiency virus: a review of the literature. J Adv Nurs 2001; 33: 357-70.

2. Ritter A, Cameron J. A review of the efficacy and effectiveness of harm reduction strategies for alcohol, tobacco and illicit drugs. Drug Alcohol Rev 2006; 25: 611-24

3. Ball AL. HIV, injecting drug use and harm reduction: a public health response. Addiction 2007; 102: 684-90.

4. Iraurgi I. La calidad de vida como indicador de resultados en la clínica de las drogodependencias. E-Boletín. Instituto para el Estudio de las Adicciones, 1999. Disponible en: http://www.ieanet.com/ boletin/ opinion.html?o=25. [Consultado 14-12-2007].

5. Fischer B, Rehm J, Kim G. Quality of Life (QoL) in Illicit Drug Addiction Treatment and Research: Concepts, Evidence and Questions. En: Von Bernd-Westermann $H$, Jellinek C, Bellmann GU. Substitution: Zwischen Leben und Sterben. Weinheim: Deutscher Studien Verlag; 2001, p. 21-40.

6. Fernández-Miranda JJ. La calidad de vida en adicciones: Una medida de la efectividad de los tratamientos. Anal Psiq 2003; 19: 377-84.

7. Bowling A. Measuring Health: A review of quality of life measurement scales. Buckingham; Open University Press; 1991. Edición en castellano: La medida de la salud. Revisión de las escalas de medida de la calidad de vida. Barcelona: Masson; 1994. 
8. Bowling A. Measuring disease. Buckingham-Philadelphia: Open University Press; 1995.

9. Bobes J, González MP, Bousoño M, editores. Calidad de vida en las esquizofrenias. Barcelona; JR Prous Editores; 1995.

10. Badia X, Salamero M, Alonso J, Ollé A. La medida de la salud. Guía de escalas de medición en español. Barcelona: PPU; 1996. / $2^{a}$ edición. Barcelona: Edimac; 1999.

11. Katschnig H, Freeman H, Sartorius N. editores. Calidad de vida en trastornos mentales. Barcelona: Masson; 2000.

12. Guyatt GH, Veldhuyzen van Zanten SJO, Feeny D, Patrick DL. Measuring quality of life in clinical trials: a taxonomy and review. Can Med Assoc J 1989; 140: 1441-8.

13. Guyatt $\mathrm{GH}$. A taxonomy of health status instruments. J Rheumat 1995; 22: 1188-90.

14. Badia X, Carné X. La evaluación de la calidad de vida en el contexto del ensayo clínico. Med Clín (Barc) 1998; 110: 550-6.

15. Iraurgi I. Instrumentos de Evaluación de la Calidad de Vida Relacionada con la Salud en Toxicomanías. En: Iraurgi I, González-Saiz F, editores. Instrumentos de evaluación en drogodependencias. Madrid: Aula Médica; 2002, p. 481-511.

16. Morales-Manrique CC, Valderrama-Zurian JC, Castellano-Gómez M, Aleixandre-Benaventa R. Medición de la calidad de vida e importancia de la atención a las necesidades autopercibidas en pacientes drogodependientes. Trast Adict 2006; 8: 212-21.

17. Wan C, Fang J, Zhang Y, He C, Chen L. Development of a Quality of Life Scale for Drug Addicts (QOL-DA). Qual Life News Lett, 1997; 17: 6.

18. Lozano O, Rojas AJ, Pérez-Meléndez C. Construcción de un Test para Medir la Calidad de Vida Relacionada con la Salud Específico para Drogodependientes. Sevilla; Dirección General para las Drogodependencias y Adicciones, Consejería para la Igualdad y Bienestar Social, Junta de Andalucía; 2006.

19. Lozano-Rojas OM, Rojas-Tejada A, Pérez-Meléndez C, Apraiz B, Sánchez-Muñoz F, Marín-Bedoya A. Test para la Evaluación de la Calidad de Vida en Adictos a Sustancias Psicoactivas (TECVASP): estudios de fiabilidad y validez. Trast Adict 2007; 9: 97-107.

20. Brogly SB, Bruneau J, Palepu A, Mercier C, Franco E. (2002). Validation of an Instrument to Measure the Quality of Life of Drug Users: The Injection Drug User Quality of Life Scale (IDUOOL). 11 th Annual Canadian Conference on HIV/AIDS Research, Winnipeg, Manitoba. Abstract in: Cannad J Infec Dis 2002; 13: Supl A.

21. Brogly SB, Mercier C, Bruneau J, Palepu A, Franco E. Towards more effective public health programming for injection drug users: Development and evaluation of the injection drug user quality of life scale. Subst Use Misuse 2003; 38: 965-92.
22. Hubley AM, Russell LB, Palepu A. Injection Drug Use Quality of Life scale (IDUOOL): A validation study. Health Qual Life Outcomes 2005; 3: 43.

23. Brogly SB, Palepu A, Franco E, Bruneau J. The Injection Drug User Quality of Life Scale (IDUQOL). 1st Canadian Network for Vaccines and Immunotherapeutics (CANVAC). Annual Scientific Meeting, Lake Louise; 2001.

24. Brogly SB, Palepu A, Franco E, Bruneau J. Development of an instrument to measure the quality of life of injection drug users: the Injection Drug User Quality of Life Scale (IDUQOL). 10th Annual Canadian Conference on HIV/AIDS Research, Toronto. Abstract in: Cannad J Infec Dis 2001; 12: Supl B.

25. Brogly SB, Bruneau J, Palepu A, Franco E. The quality of life of Montreal injection drug users: a pursuit towards more effective public health programming. Int Conf AIDS 2002; 14: 7-12.

26. Brogly SB, Bruneau J, Palepu A, Franco E. Quality of Life and Public Health Program Use of Montreal Cocaine and Heroin Injection Drug Users. $12^{\text {th }}$ Annual Canadian Conference on HIV/AIDS Research, Halifax, Nova Scotia. Abstract in: Cannad J Infec Dis 2003; 14: Supl A.

27. Hattie J. Methodology review: Assessing unidimensionality of tests and items. Appl Psych Meas 1984; 20: 1-14.

28. Hattie J. An empirical study of the various indices for determining unidimensionality. Multivar Behav Res 1985; 19: 49-78.

29. Gorsuch RL. Factor analysis. Hillsdale, NJ: Lawrence Erlbaum; 1983.

30. Darke S, Hall W, Wodak A, Heather N, Ward J. Development and validation of a multidimensional instrument for assessing outcome of treatment among opiate users: the Opiate Treatment Index. Brit J Addict 1992; 87: 733-42.

31. González-Saiz F, Salvador-Carulla L, Martínez-Delgado JM, López-Cárdenas A, Ruz-Franzi I. Estudio de validez y fiabilidad de la versión española de la Entrevista Clínica Opiate Treatment Index (OTI). En: Iraurgi I, González-Saiz F, editores. Instrumentos de evaluación en drogodependencias. Madrid: Aula Médica; 2002, p. 309-59.

32. McLellan AT, Kushner H, Metzger D, Peters R, Smith I, Grissom G, Pettinati $H$, Argeriou M. The fifth edition of the Addiction Severity Index. J Subs Abuse Treat 1992; 9: 199-213.

33. González-Saiz F, Salvador-Carulla L, Martínez-Delgado JM, López-Cárdenas A, Ruz-Franzi I, Guerra-Díaz D. Estudio de fiabilidad y validez de la versión española de la Entrevista Clínica Addiction Severity Index (ASI). En: Iraurgi I, González-Saiz F. editores. Instrumentos de evaluación en drogodependencias. Madrid: Aula Médica; 2002, p. 271-307.

34. Ware JE, Sherbourne CD. The MOS 36-item short form health survey (SF-36): Conceptual framework and item selection. Med Care 1992; 30: 473-83. 
35. Ware JE, Snow KK, Kosinski M, Gandek B. SF-36 Health Survey. Manual and Interpretation Guide. Boston, MA: The Health Institute, New England Medical Center; 1993.

36. Alonso J, Prieto L, Antó JM. La versión española del SF-36 Health Survey (Cuestionario de Salud SF-36): un instrumento para la medida de los resultado clínicos. Med Clin (Barc) 1995; 104: 771-6.

37. Alonso J, Regidor E, Barrio G, Prieto L, Rodríguez C, de-la-Fuente L. Valores poblacionales de referencia de la versión española del Cuestionario de Salud SF-36. Med Clin (Barc) 1998; 111: 410-6.

38. Gandek B, Ware JE. Translating functional health and well-being: International Quality of Life Assessment (IOOLA) Project studies of the SF-36 Health Survey. J Clin Epidemiol 1998; 51: 891-1214.

39. IMIN - Institut Municipal d'Investigació Mèdica. Manual de puntuación de la versión española del Cuestionario de Salud SF-36. Disponible en: http://www.imim.es/ imim/cas/c-QOV.htm [Consultado 14-04-2007].

40. EuroQol Group. EuroQol - A new facility for the measurement of health-related quality of life. Health Policy 1990; 16: 199-208.

41. Badía X, Roset M, Montserrat S, Herdman M, Segura A. La versión española del EuroQol: descripción y aplicaciones. Med Clin (Barc) 1999; 112, Supl 1: 79-96.

42. Prieto L, Sacristán J. What is the value of social values? The uselessness of assessing health-related quality of life through preference measures. BMC Med Res Methodology 2004; 4: 10.

43. Pett MA, Lackey NR, Sullivan JJ. Making sense of factor analysis: The use of factor analysis for instrument development in health care research. Thousand Oaks, CA: SAGE Publications; 2003.

44. Bentler PM, Wu EC. EQS for Windows User's Guide. Encino, CA: Multivariate Software Inc; 1995.

45. Bentler PM, Bonett DG. Significant test and goodness of fit in the analysis of covariances structures. Psychol Bull 1980; 88: 588-606.

46. Marsch HW, Balla J, McDonald RP. Goodness of fit in confirmatory factor analysis: the effect of sample size. Psychol Bull 1988; 103: 391-410.

47. Clark LA, Watson D. Constructing validity: Basic issues in objective scale development. Psychol Assessment 1995; 7: 309-19.

48. Nunnally JC, Berstein IJ. Teoría psicométrica. $3^{a}$ Edición. México: McGraw-Hill; 1995.

49. Messick S. Validity of test interpretation and use. Research Report No. 90-11. Educational Testing Service; 1990.

50. Foster SL, Cone JD. Validity issues in clinical assessment. Psychol Assessment 1995; 7: 248-60.

51. Hair JF, Anderson RE, Tatham RL, Black WC. Análisis Multivariante. $5^{a}$ edición. Prentice Hall International, Madrid; 1999.

52. Morales-Manrique CC, Castellano-Gómez M, ValderramaZurian JC, Aleixandre-Benaventa R, Papelu A, and Cocaine Group. Cross cultural adaptation of the Injection Drug User Quality Of Life Scale (IDUQOL) in Spanish drug dependent population, with or without injectable consumption: Quality of Life Scale-Spanish (DUQOLSpanish). Addic Behav 2007; 32: 1913-21. 